\title{
Mesoscopic Fano Effect in Aharonov-Bohm Rings with an Embedded Double Dot
}

\author{
B. Tanatar*, V. Moldoveanu ${ }^{\dagger}$, M. Ţolea ${ }^{\dagger}$ and A. Aldea ${ }^{\dagger}$ \\ ${ }^{*}$ Department of Physics, Bilkent University, Bilkent, 06800 Ankara, Turkey \\ ${ }^{\dagger}$ National Institute of Materials Physics, P.O. Box MG-7, Bucharest-Magurele, Romania
}

\begin{abstract}
We investigate theoretically in a tight-binding model the transport properties of the Aharonov-Bohm interferometer (ABI) with one dot embedded in each of its arms. For weak interdot coupling the model Hamiltonian describes the system considered in the experiments of Holleitner et al. [Phys. Rev. Lett. 87, 256802 (2001)]. The electronic transmittance of the interferometer is computed within the Landauer-Büttiker formalism while the coexistence of resonant and coherent transport is explicitly emphasized by using the Feschbach formula. The latter produces effective Hamiltonians whose spectral properties describe the tunneling processes through each dot. We reproduce numerically the stability charging diagrams reported in the experiments of Holleitner et al. When the magnetic flux is fixed and one dot is set to resonance the interferometer transmittance shows Fano lineshapes as a function of the gate voltage applied to the other dot. Our model includes the effect of the magnetic field on the dot levels and explains the change of the asymmetric tail as the magnetic flux is varied. The transmittance assigned to the Fano dips located in the almost crossing point of the charging diagrams shows Aharonov-Bohm oscillations.
\end{abstract}

Keywords: Quantum dots, Aharonov-Bohm interferometer, Fano effect PACS: 73.23.Hk, 85.35.Ds, 85.35.Be, 73.21.La

\section{GENERAL FRAMEWORK}

The Aharonov-Bohm interferometers are hybrid systems composed of one or several quantum dots embedded in the arms of a mesoscopic ring. The interferometer Hamiltonian $H^{I}$ acts on $\mathscr{H}^{I}=\mathscr{H}^{R} \oplus \mathscr{H}^{C}$, where $\mathscr{H}^{C}$ and $\mathscr{H}^{R}$ are the Hilbert spaces of the quantum dot cluster and the truncated ring. The latter is coupled to several semi-infinite noninteracting leads labelled $\alpha, \beta \ldots . H^{I}$ is conveniently written as:

$$
\begin{aligned}
H^{I} & =H^{C}+H^{R}+H^{C R}+H^{R C} \\
H^{C R}+H^{R C} & =\tau \sum_{m}\left(e^{-i \varphi_{m}}|m\rangle\langle 0 m|+h . c\right) .
\end{aligned}
$$

The off-diagonal parts $H^{C R}, H^{R C}$ connect the two subsytems, $\tau$ being the ring-dot coupling constant. $m$ is the site of the cluster that is the closest one to the site $0 \mathrm{~m}$ of the truncated ring. $H^{C}$ contains a sum of single-dot terms $H^{D_{k}}$ and their couplings:

$$
H^{D_{k}}=-e V_{k} \sum_{i \in Q D_{k}}|i\rangle\left\langle i\left|+t_{D} \sum_{<i, i^{\prime}>} e^{2 \pi i \varphi_{i i^{\prime}}}\right| i\right\rangle\left\langle i^{\prime}\right|
$$

The on-site term $V_{k}$ simulates the gate potential applied to the $\operatorname{dot} k<i, i^{\prime}>$ denotes the nearest neighbor summation and $t_{D}$ is the hopping integral on dots. The magnetic flux $\phi$ is described through Peierls phases and will be expressed in units of quantum flux $\Phi_{0}$. The conductance matrix $G$ can be computed from the Landauer-Büttiker formula provided one knows an effective Green function $G_{\text {eff }}^{I}$ of the sample in the presence of the leads. If the leads are weakly coupled the transport through the sample is easily studied by looking at the complex poles of $G_{\text {eff }}^{I}$ $[1,2]$. This situation is different in the experiments with $\mathrm{ABI}$ because the weak-coupling is set between the ring and the dot cluster while the electrons from leads reach freely the interferometer. Moreover, the complexity of the system yields complicated contributions to transport which have to be discerned at the level of the effective Green function. The remedy is to use the Feschbach formula (see [2] for details) to express the effective resolvent in the following form:

$$
G_{\text {eff }}^{I}=\left(\begin{array}{cc}
G^{C} & -G^{C} H^{C R} G^{R} \\
-G^{R} H^{R C} G^{C} & G^{R}+G^{R} H^{R C} G^{C} H^{C R} G^{R}
\end{array}\right)
$$

The new effective Green functions $G^{C}$ and $G^{R}$ describe individually the dot and the truncated ring:

$$
\begin{aligned}
& G^{R}(z):=\left(H^{R}-\Sigma^{L}(z)-z\right)^{-1} \\
& G^{C}(z):=\left(H^{D}-\Sigma^{C}(z)-z\right)^{-1} .
\end{aligned}
$$

$\Sigma^{L}(z)$ is the lead's self-energy and the cluser self-energy $\Sigma^{C}(z)=H^{C R}\left(Q H_{\text {eff }}^{I} Q-z\right)^{-1} H^{R C}$ where $Q$ projects on the Hilbert space of the truncated ring. The conductance across the interferometer is given by

$g_{\alpha \beta}\left(E_{F}\right)=4 \tau^{4} \sin ^{2} k\left|\sum_{m, n} e^{i\left(\varphi_{m}-\varphi_{n}\right)} G_{\alpha, 0 m}^{R} G_{m n}^{C} G_{0 n, \beta}^{R}\right|^{2}$.

This formula captures all the resonant processes inside the interferometer. Our method involves only Green functions is an alternative to the scattering theoretical approach [3]. 


\section{FANO EFFECT IN DOUBLE-DOT INTERFEROMETER}

In this section we concentrate on Eq. (7) in which a double-dot interferometer is characterized. Thus $H^{C}=$ $H^{\mathrm{QD}_{1}}+H^{\mathrm{QD}_{2}}+H^{\text {tun }}$, the last term describing the interdot tunnel coupling. Clearly, the condition for quantum interference is that both dots transmit. This means that the electron tunnels simultaneuosly through two levels of the isolated double dot system $E_{i}\left(V_{1}, V_{2}\right)$ and $E_{j}\left(V_{1}, V_{2}\right)$. Here $V_{1}, V_{2}$ are the gate potentials applied on each dot. Following Holleitner et al. [4] we plot in Fig. 1 the calculated charging diagram of an interferometer with $4 \times 5$ sites noninteracting quantum dots in the weak coupling regime. For each fixed value of $V_{2}$, we varied $V_{1}$ in the interval shown in the figures and we selected only conductances $g_{12}$ that are larger than 0.65 , which means that what we obtain is roughly a map for the peak positions in the plane $\left(V_{1}, V_{2}\right)$. Each horizontal (vertical) trace represents the trajectory of a conductance peak associated with a resonant tunneling process through $\mathrm{QD}_{2}\left(\mathrm{QD}_{1}\right)$.

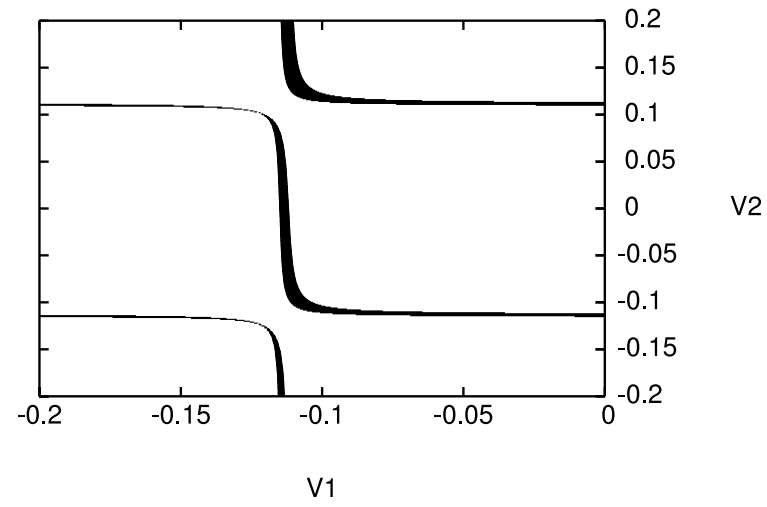

FIGURE 1. Charging diagram of the double dot interferometer with ring-dot coupling constant $\tau=0.3$ and $\phi=3 \Phi_{0}$.

The idea is then to isolate the resonant contribution of a pair of eigenvalues in the effective resolvent. To this end one has first to use again the Feschbach formula in order to single out an effective resolvent acting in the two-dimensional spectral subspace of the two chosen eigenvalues. As a consequence, $G^{C}$ is approximated by a $2 \times 2$ matrix $\tilde{G}_{\text {eff }}^{D}$. Secondly, a Dyson equation for $\tilde{G}_{\text {eff }}^{C}$ is written down, with respect to its off-diagonal part. The unperturbed resolvent involved in the Dyson expansion is the sum of two Breit-Wigner-like terms associated with the resonances located near $E_{i}$ and $E_{j}$. By plugging the Dyson expansion for $\tilde{G}_{\text {eff }}^{D}$ in (7) one recovers all the electronic paths within the interferometer. More technical details were given in [2]. In Fig. 2 we show a detail from the charging diagram in Fig. 1, taken in the neighborhood of an almost crossing point. We observe

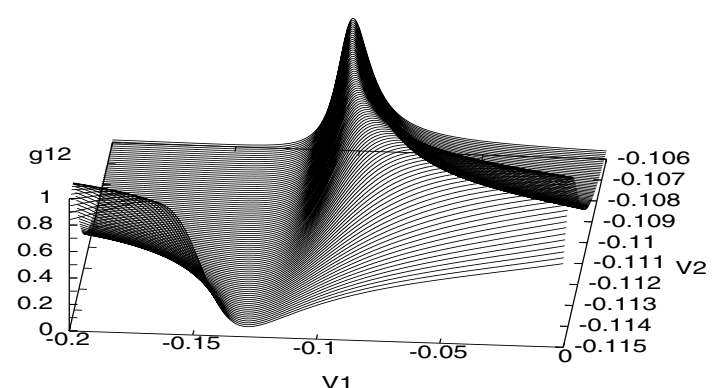

FIGURE 2. Fano effect in the double-dot interferometer.

an asymmetric large tail of the peaks, showing clearly that in this regime the interferometer acts as a Fano system. This happens because one dot $\left(\mathrm{QD}_{2}\right)$ is always set to a resonance thus the corresponding arm of the ring is 'free', providing the continuum component for the interference. As $V_{2}$ is slightly modified the orientation of the Fano tail changes. This is the so-called electrostatic control of the Fano interference [5]. Moreover, the transmittance assigned to the Fano dips shows Aharonov-Bohm oscillations, in full agreement with the observations of Holleitner et al. These results were thoroughly discussed in [2].

\section{ACKNOWLEDGMENTS}

B. T. thanks the Turkish Academy of Sciences (TUBA) for partial support. V. M. thanks the Scientific and Technical Research Council of Turkey (TUBITAK) for partial support.

\section{REFERENCES}

1. S. Datta, Electronic Transport in Mesoscopic Systems, Cambridge: Cambridge University Press, 1995, pp. 132-174.

2. V. Moldoveanu, M. Tolea, A. Aldea, and B. Tanatar, Phys. Rev. B 71, 125338 (2005).

3. G. Hackenbroich and H. A. Weidenmüller, Phys. Rev. B 53, 16379 (1996).

4. A. W. Holleitner, C. R. Decker, H. Qin, K. Eberl, and R. H. Blick, Phys. Rev. Lett. 87, 256802 (2001).

5. K. Kobayashi, H. Aikawa, S. Katsumoto, and Y. Iye, Phys. Rev. Lett. 88, 256806 (2002); Phys. Rev. B 68, 235304 (2003). 